Research Article

\title{
Selection Theory of Dendritic Growth with Anisotropic Diffusion
}

\author{
Martin von Kurnatowski and Klaus Kassner \\ Institut für Theoretische Physik, Otto-von-Guericke-Universität Magdeburg, Postfach 4120, 39016 Magdeburg, Germany \\ Correspondence should be addressed to Klaus Kassner; klaus.kassner@ovgu.de
}

Received 29 October 2014; Accepted 21 December 2014

Academic Editor: Ivan Smalyukh

Copyright (C) 2015 M. von Kurnatowski and K. Kassner. This is an open access article distributed under the Creative Commons Attribution License, which permits unrestricted use, distribution, and reproduction in any medium, provided the original work is properly cited.

\begin{abstract}
Dendritic patterns frequently arise when a crystal grows into its own undercooled melt. Latent heat released at the two-phase boundary is removed by some transport mechanism, and often the problem can be described by a simple diffusion model. Its analytic solution is based on a perturbation expansion about the case without capillary effects. The length scale of the pattern is determined by anisotropic surface tension, which provides the mechanism for stabilizing the dendrite. In the case of liquid crystals, diffusion can be anisotropic too. Growth is faster in the direction of less efficient heat transport (inverted growth). Any physical solution should include this feature. A simple spatial rescaling is used to reduce the bulk equation in $2 \mathrm{D}$ to the case of isotropic diffusion. Subsequently, an eigenvalue problem for the growth mode results from the interface conditions. The eigenvalue is calculated numerically and the selection problem of dendritic growth with anisotropic diffusion is solved. The length scale is predicted and a quantitative description of the inverted growth phenomenon is given. It is found that anisotropic diffusion cannot take the stabilizing role of anisotropic surface tension.
\end{abstract}

\section{Introduction}

Dendritic microstructures develop from an interface instability of a crystal growing into its melt. In the simplest models, the growth process is driven solely by thermal diffusion. The liquid has to be cooled externally to keep the system out of equilibrium. Neglecting surface tension, Ivantsov found a continuous family of unstable solutions corresponding to parabolic dendrites with isothermal interfaces [1]. Out of this family, only a discrete set containing a single linearly stable solution survives, if anisotropic surface tension is taken into account. Hence, anisotropic surface tension acts as a singular perturbation. The selected solution is the stable one [2]. The methodology leading to these results is also referred to as "microscopic solvability theory" (MST). It is in good agreement with experiments for substances with weak capillary anisotropy such as ammonium bromide solutions [3], whereas for other materials such as pivalic acid the predictions appear to be less accurate $[4,5]$.
In view of this situation, the consideration of additional effects seems a worthwhile effort. Recently, the presence of grain boundaries was successfully included into the theory [6]. Furthermore, the selection problem was treated for convective systems, where the flow introduces additional complexity [7-9]. Interfacial patterns have also been observed in liquid crystals at nematic-isotropic boundaries $[10,11]$, as well as at nematic-smectic- $B$ boundaries $[12,13]$. In these materials, the thermal diffusivity can depend significantly on the spatial direction. The special role of anisotropy in MST gives rise to the question whether the presence of anisotropic diffusion can by itself lead to stable growth modes.

The focus of this work is on the two-dimensional case of a smectic-B liquid crystal, growing into its own undercooled nematic phase as considered by Börzsönyi et al. [13]. In the experiments, the smectic- $B$ phase is aligned homeotropically; that is, its director is perpendicular to the growth plane, whereas the nematic director $\vec{n}_{n}$ is in-plane (see Figure 1). Thermal diffusion in the nematic phase is least 


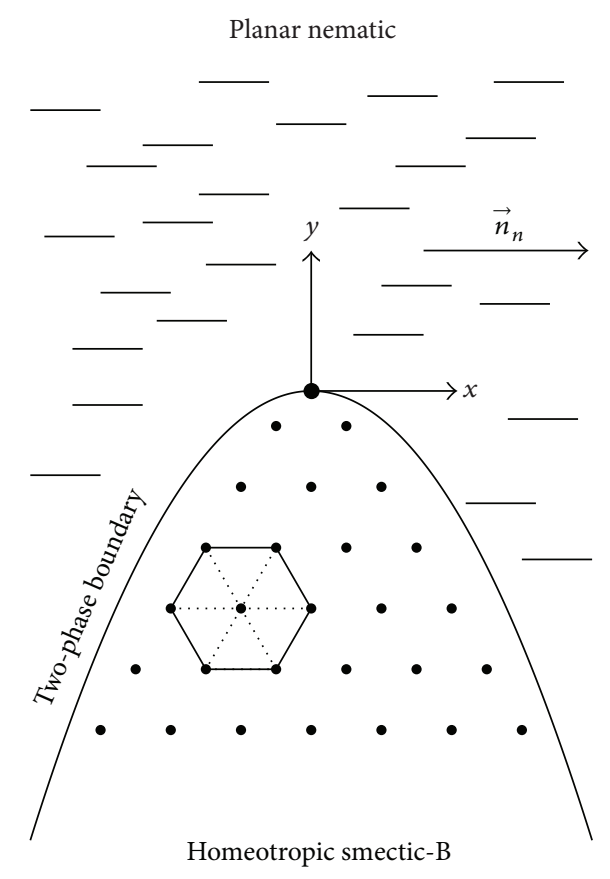

FIGURE 1: Scheme of the liquid crystal system setup considered here.

efficient orthogonally to the nematic director. This is the growth direction chosen by the crystal. Experiments show that the normal growth velocity along the interface takes its largest value in the direction of lowest thermal diffusivity (inverted growth) $[12,13]$. The phenomenon is somewhat counterintuitive, because diffusion removes the latent heat from the interface and enables steady-state dendritic growth, so one might a priori expect growth to proceed preferentially along directions of fast diffusion. This is not the case, which may be understood from realizing that globally heat extraction is most efficient, if diffusion is fast perpendicular to the extended flanks of the crystal instead of being fast perpendicular to the smallish tip. Any realistic solution must of course reproduce this feature.

We solve the selection problem of diffusion-limited dendritic growth with anisotropic heat transport by means of the one-sided model of solidification. In Section 2, the model equations are introduced and transformed to dimensionless quantities. A family of (unstable) solutions in the absence of surface tension is derived in Section 3. Singular perturbation theory starting from this set of solutions yields a local eigenvalue equation for the selected growth mode. Its numerical solution is exhibited in Section 4. The existence of a solution without anisotropy of surface tension is investigated, and a new scaling law for the stability parameter as a function of the heat diffusion anisotropy strength is given. Our main findings are summarized in Section 5.

\section{Growth Model}

The diffusion equation

$$
\nabla \cdot(\widehat{D} \cdot \nabla T)=\frac{\partial T}{\partial t}
$$

is used to describe heat transport, and the thermal diffusivity

$$
\widehat{D}=\left(\begin{array}{cc}
D & 0 \\
0 & \mu^{2} D
\end{array}\right)
$$

has to be given as a second-rank tensor. The dimensionless positive number $\mu \in(0,1)$ is the heat diffusion anisotropy strength. $\mu$ is smaller than one here, which means that heat transport along the $y$ direction is less efficient. For $\mu \rightarrow 1$, the isotropic diffusion limit is attained.

Heat transport in the smectic-B phase does not have the same anisotropy as in the nematic phase. Strictly speaking, that anisotropy is sixfold due to the smectic-B lattice structure, in contrast to the twofold anisotropy in the nematic phase. Especially in the case of a homeotropic smectic, the anisotropy may be assumed to be much weaker than in the nematic phase or even absent. Consequently, $\widehat{D}$ cannot be assumed to be equal in the smectic- $B$ domain and in the nematic domain, and the two phases cannot be treated symmetrically. However, in experiments the smectic-B liquid crystal remains nearly isothermal during the whole growth process [13]. Thus, thermal diffusion in the smectic-B domain can only play a marginal role. This argument suggests the applicability of a one-sided model, which significantly simplifies the problem mathematically compared to an approach with two different diffusion tensors. Hence, the field equation (1) will only be solved in the nematic phase.

The boundary conditions

$$
\begin{aligned}
& \left.T\right|_{y_{s}}=T_{M}-\frac{L}{c} d_{0} \kappa a(\theta), \\
& -(\widehat{D} \cdot \nabla T)_{y_{s}} \cdot \vec{n}=\frac{L}{c} \vec{V} \cdot \vec{n}
\end{aligned}
$$

must hold on the interface designated by $y_{s}(x, t)$. Here, $T_{M}$ is the equilibrium melting temperature, $d_{0}$ is the capillary length, which is proportional to the orientational average $\gamma_{0}$ of the surface tension, $\kappa$ is the curvature, and $a(\theta)$ is the surface stiffness anisotropy function $\left[a(\theta)=\left(\gamma(\theta)+\gamma^{\prime \prime}(\theta)\right) / \gamma_{0}\right.$ with $\gamma(\theta)$ the orientation-dependent surface tension]. $\theta$ indicates the orientation, being defined as the angle between the interface normal and a fixed direction (the growth direction along the $y$-axis in this case). $L$ is the latent heat, which has to be transported away from the two-phase boundary as expressed by (1). $\vec{V}$ is the growth velocity, $c$ is the specific heat per unit volume, and $\vec{n}=\left(\vec{e}_{y}-y_{s}^{\prime} \vec{e}_{x}\right) / \sqrt{1+\left(y_{s}^{\prime}\right)^{2}}$ is the interface unit normal vector pointing into the nematic phase. Assuming local equilibrium at the two-phase boundary, the Gibbs-Thomson condition (3) describes the dependence of the interface temperature on its local shape. For growth perpendicular to the nematic director, the kinetics of molecule incorporation into the smectic-B crystal involves mainly twist. Hence, kinetic effects are rather fast, so they may be neglected and only the capillary term is included. This constitutes a considerable simplification. Otherwise, there would be a velocity-dependent term in (3). The additional condition (4) is required, because the determination of the interface shape and position $y_{s}(x, t)$ at time $t$ is part of 
the problem. The condition ensures heat conservation at the phase transition. It is sometimes also referred to as Stefan condition. Far away from the two-phase boundary, the temperature must take a fixed value $T_{\infty}$ determined by the external cooling:

$$
\lim _{|\vec{r}| \rightarrow \infty} T=T_{\infty}
$$

In order to obtain dimensionless equations, the temperature is written as $T \rightarrow T_{M}+(L / c) T$, and all lengths are measured in units of the tip curvature radius $\rho$. We look for stationary solutions in a moving frame of reference attached to the dendrite tip; that is, we set the growth velocity vector $\vec{V}=V \vec{e}_{y}$ and $\vec{r} \rightarrow \vec{r}+V t \vec{e}_{y}$. A simple but essential step is the spatial rescaling of the $y$-axis:

$$
y=\mu \bar{y}
$$

Using $\partial / \partial y=(1 / \mu)(\partial / \partial \bar{y})$ and dropping the bar over $y$ immediately, the transformed diffusion equation reads

$$
T_{x x}+T_{y y}+P_{\mu} T_{y}=0
$$

and the interface conditions (3) and (4) become

$$
\begin{gathered}
\left.T\right|_{y_{s}}=-\frac{1}{2} \sigma \bar{\kappa} a(\theta), \\
-\left(T_{y}-y_{s}^{\prime} T_{x}\right)_{y_{s}}=1 .
\end{gathered}
$$

Here, the interface position is denoted by $y_{s}(x) . \sigma=2 d_{0} / \rho P_{\mu}$ is the stability parameter and the dimensionless temperature is measured in units of the effective growth Péclet number $P_{\mu}=\rho V / \mu D$. The prime in (9) denotes the total derivative with respect to $x$. The far-field boundary condition (5) reads

$$
\lim _{|\vec{r}| \rightarrow \infty} T=-\frac{\Delta}{P_{\mu}}
$$

with the dimensionless undercooling $\Delta=c\left(T_{M}-T_{\infty}\right) / L$.

The mathematical structure of problem (7), (8), (9), and (10) is almost the same as in the case of isotropic diffusion [2]. The difference lies in $a(\theta)$ and in the function

$$
\bar{\kappa}=-\frac{\mu y_{s}^{\prime \prime}}{\left(1+\mu^{2} y_{s}^{\prime 2}\right)^{3 / 2}},
$$

which is not the interface curvature anymore in the rescaled coordinate system. Since these terms, appearing in (8), drop out of the problem for $\sigma=0$, rendering the isotropic and anisotropic diffusion cases mathematically equivalent, there can be no stable solution in the absence of surface tension.

\section{Growth Mode Selection}

In the case of vanishing surface tension $(\sigma=0)$, the twophase boundary is located at $y_{s}^{0}(x)=(1 / 2)\left(1-x^{2}\right)$ and it is isothermal and parabolic. The corresponding solution to (7) is

$$
\begin{aligned}
T^{0}(x, y) \\
=-\sqrt{\frac{\pi}{2 P_{\mu}} e^{P_{\mu} / 2}} \\
\quad \times\left[\operatorname{erfc}\left(\sqrt{\frac{P_{\mu}}{2}}\right)-\operatorname{erfc}\left(\sqrt{\frac{P_{\mu}}{2}} \sqrt{y+\sqrt{x^{2}+y^{2}}}\right)\right]
\end{aligned}
$$

with erfc() denoting the complementary error function. It fulfills the interface conditions $T^{0}\left(x, y_{s}^{0}\right)=0$ (derived from (8)) and $-1=T_{y}^{0}\left(x, y_{s}^{0}\right)+x T_{x}^{0}\left(x, y_{s}^{0}\right)$ (derived from (9)). Inserting solution (12) into (10) yields the condition

$$
\Delta=\sqrt{\frac{\pi P_{\mu}}{2}} e^{P_{\mu} / 2} \operatorname{erfc}\left(\sqrt{\frac{P_{\mu}}{2}}\right),
$$

from which a value for $P_{\mu}$ can be calculated for given undercooling. However, it is not possible to calculate separate values of $V$ and $\rho$ from (13). Moreover, Ivantsov's solutions are unstable for isotropic diffusion and this must be the case here too, because, for $\sigma=0$, the original and rescaled models are identical. A stable solution can only be found, if surface tension is taken into account; that is, $\sigma$ is small but nonzero. $T$ is expanded about solution (12) of the unperturbed problem: $T \rightarrow T^{0}+T$, and the interface position is written as $y_{s}(x)=$ $y_{s}^{0}(x)+\zeta(x)$. Equation (7) also applies to the temperature correction $T$, because it is a linear equation, and in the limit $P_{\mu} \rightarrow 0$ it can be replaced by

$$
T_{y}=i T_{x}
$$

close to the singularity at $x=-i / \mu$ of $(8)$ in the complex plane [8]. The interface conditions (8) and (9) are expanded about $y_{s}^{0}$ :

$$
\begin{gathered}
\left.T\right|_{y_{s}^{0}}=-\frac{1}{2} \sigma \bar{\kappa} a(\theta)+\frac{\zeta(x)}{1+x^{2}}, \\
-\left(T_{y}-y_{s}^{\prime} T_{x}\right)_{y_{s}^{0}}=\left[\frac{x \zeta(x)}{1+x^{2}}\right]^{\prime} .
\end{gathered}
$$

In condition (16), a nonlinear term $\mathscr{O}\left(\zeta \zeta^{\prime}\right)$ was dropped. It will turn out later that this neglect is indeed justified. Equation (14) is inserted into the interface conditions (15) and (16). When taking the total derivative of (15), we have to differentiate along the interface; that is, $d / d x=(\partial / \partial x)+$ $y_{s}^{\prime}(\partial / \partial y)$. Some simple manipulations of the resulting linear equation system allow complete elimination of $T_{x, y}$, and one finds

$$
\frac{1}{2} \sigma \bar{\kappa} a(\theta)=\frac{1-i x}{1+x^{2}} \zeta(x)
$$

determining the shape correction function $\zeta(x)$. This is an eigenvalue problem for the stability parameter $\sigma$. The underlying model was reduced to the case of isotropic diffusion 
with the exception of $\bar{\kappa}$ and $a(\theta)$, which did not have to be inserted until now. Thus, (17) could have also been obtained by following the rigorous classical solution scheme [14], neglecting some nondivergent terms. Here, it was derived in a much simpler manner by using (14). As explained in [15], this leads to the same results as the classical methods. The factor $1 / 2$ in front of $\sigma$ does not occur in the symmetric model but is expected here [16].

Equation (17) is transformed onto a small disk around the singular point $-i / \mu$ of the problem in the complex plane, and the corresponding solution must asymptotically match a solution far from the singularity. The scaling transformation $x=(-i / \mu)\left(1-\bar{\sigma}^{\alpha} t\right), \zeta(x)=\bar{\sigma}^{2 \alpha} \phi(t)$ with $\bar{\sigma}=(1 / 2) \sigma$ is applied to (17). Here, $\alpha$ is a scaling exponent to be found. We could assume a fourfold anisotropy $a(\theta)=1-\beta_{4} \cos (4 \theta)$ of surface stiffness. But here it is particularly reasonable to use a twofold anisotropy $a(\theta)=1-\left(\beta_{2} / 2\right) \cos (2 \theta)$ of surface tension, because the twofold term is the dominant contribution in the considered system of a homeotropic smectic-B in a planar nematic [13]. One finds

$$
\frac{1+\mu^{2} \ddot{\phi}}{(2 \tau)^{3 / 2}}\left[1-\frac{b_{2}}{2 \tau}\right]=\frac{\phi}{1+\mu}
$$

with $\tau=\mu^{2} \dot{\phi}+t$. The exponent was set as $\alpha=2 / 7$ in order to obtain the same leading orders on both sides. This is the same scaling exponent as in the case of isotropic diffusion [14]. $\zeta^{\prime \prime}$ is of the order $\mathcal{O}(1)$, but $\zeta^{\prime}$ is indeed of the order $\bar{\sigma}^{\alpha}$. This justifies the linearization in condition (16). It also ensures that $\bar{\kappa}$ and $a(\theta)$ diverge in the limit $\sigma \rightarrow 0$, so the term $\sigma \bar{\kappa} a(\theta)$ remains large enough to permit its being decisive in selection. The nonlinear eigenvalue $b_{2}=\beta_{2} / \bar{\sigma}^{2 / 7}$ remains the only free parameter group aside from $\mu$. The factor in square brackets on the left-hand side is the transformed version of $a(\theta)$. Only leading terms of $\bar{\sigma}^{\alpha}$ were kept in (18). It should be kept in mind that the stability parameter $\sigma$ has twice the value of $\bar{\sigma}: \sigma=2 \bar{\sigma}=2 \times\left(\beta_{2} / b_{2}\right)^{7 / 2} . \bar{\sigma}$ would be the right effective stability parameter, if the symmetric model was used. Since $b_{2}$ can be assumed to be of the order $\mathcal{O}(1)$, we have $\sigma \propto \beta_{2}^{7 / 2}$. This can be generalized for an $n$-fold anisotropy of surface tension: $\sigma \propto \beta_{n}^{7 / n}$. An explicit formula (19) for the growth velocity $V$ can be given:

$$
V=\frac{2 D \Delta^{4} \mu \sigma(\mu)}{\pi^{2} d_{0}} .
$$

Equation (19) holds for small Péclet numbers $P_{\mu}$. The dependence on the undercooling is the same as in the case of isotropic heat transport, but there is an additional factor $\mu$.

In fact, (18) can be converted into an equation having the form of the local equation from the corresponding problem with isotropic diffusion. This is achieved by rescaling: $t=$ $\mu^{4 / 7}((1+\mu) / 2)^{2 / 7} \widetilde{t}, \phi(t)=\mu^{-6 / 7}((1+\mu) / 2)^{4 / 7} \Psi(\widetilde{t})$. The inner equation becomes

$$
\frac{1+\Psi^{\prime \prime}}{\left(2 \widetilde{\tau}_{\mu}\right)^{3 / 2}}\left(1-\frac{\widetilde{b}_{2}}{2 \widetilde{\tau}_{\mu}}\right)=\frac{\Psi}{2}
$$

with $\tilde{\tau}_{\mu}=\tilde{t}+\Psi^{\prime}$, and the nonlinear eigenvalue $\tilde{b}_{2}=$ $\left(\beta_{2} / \bar{\sigma}^{2 / 7}\right)\left[2 / \mu^{2}(1+\mu)\right]^{2 / 7}$ is expressed as a function of $\bar{\sigma}$ and $\mu$. Here, the prime denotes the derivative with respect to $\tilde{t}$. Equation (20) is equivalent to (18) at $\mu=1$. Only the respective eigenvalues $\widetilde{b}_{2}$ and $b_{2}$ are defined differently. Using the definitions of $\tilde{b}_{2}, \bar{\sigma}$, and $P_{\mu}$, one finds the analytical formulas

$$
\begin{aligned}
& V=\left(\frac{\beta_{2}}{\widetilde{b}_{2}}\right)^{7 / 2} \frac{P_{\mu}^{2} D}{d_{0}} \frac{2}{\mu(1+\mu)}, \\
& \rho=\left(\frac{\beta_{2}}{\widetilde{b}_{2}}\right)^{-7 / 2} \frac{d_{0}}{P_{\mu}} \frac{\mu^{2}(1+\mu)}{2},
\end{aligned}
$$

for $V$ and $\rho$, which can be evaluated once a value for $\widetilde{b}_{2}$ is obtained numerically.

\section{Numerical Results}

For given values of $\Delta, \beta_{2}$, and $\mu$, the set (13), (20) is solved numerically. For details about the numerical method, see [9, 17]. Explicit results are shown for the substance $\mathrm{CCH} 5$ (4-npentyl- $4^{\prime}$-cyano-trans 1,1 -bicyclohexane, $T_{M}=51.2^{\circ} \mathrm{C}[18]$, $\beta_{2}=0.06 \cdots 0.18$ [13], $\mu=0.767, D=1.25 \cdot 10^{5} \mu \mathrm{m}^{2} / \mathrm{s}$, and $\left.d_{0}=5 \cdot 10^{-6} \mu \mathrm{m}\right)$. It is a long organic molecule consisting of a pentyl chain, two cyclohexane rings, and a nitrile group. The values of $\mu$ and $D$ were measured for the substance K15 ( $4^{\prime}$-n-pentyl-4-cyanobiphenyl) in [19].

But they can be assumed to be similar for $\mathrm{CCH} 5$, because they depend almost only on the alkyl chain length [18], which is equal for both substances. The two molecules are depicted in Figure 2. Moreover, the value of $d_{0}$ is only a rough estimate for nematic-smectic interfaces derived from the surface tension measurements of Buka et al. [20].

From (20), we find the lowest eigenvalue $\widetilde{b}_{2}=1.6608$, corresponding to the selected growth state. The fourfold surface tension anisotropy version of (18) was also implemented yielding $b_{4}=\beta_{4} / \bar{\sigma}^{4 / 7}=0.6122$ at $\mu=1$. This value was also found by Brener and Mel'nikov [14] and Tanveer [17].

Figure 3 reveals the dependence of the observable quantities on the thermal diffusion anisotropy strength $\mu$ at different values of the dimensionless undercooling $\Delta$. These are plots of the functions from (21) and (22). $\mu$ is tunable to a limited amount even in experiments by using substances with different alkyl chain lengths. The lowest undercooling in use here is $\Delta=0.02$ corresponding to an absolute value of about $0.23 \mathrm{~K}$ for CCH5. Börzsönyi et al. [13] observed the onset of the dendritic growth regime already at undercoolings larger than about $0.15 \mathrm{~K}$. Thus, we are in the experimentally relevant range. At the largest undercooling $\Delta=0.04$ used here, the effective growth Péclet number $P_{\mu}$ still hardly exceeds $10^{-3}$. The largest possible value $\beta_{2}=0.18$ consistent with experiments for CCH5 [13] was used. The growth velocity $V$ is a monotonically decreasing function of $\mu$, which may be regarded as a full quantitative description of the "inverted growth" phenomenon observed, for instance, in [12]. The relevant direction for heat transport is perpendicular to 


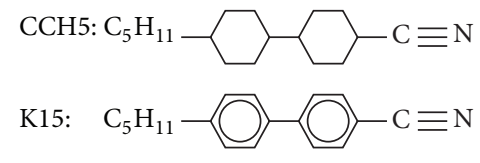

FIGURE 2: Structure formulas of the liquid crystal molecules CCH5 and K15.

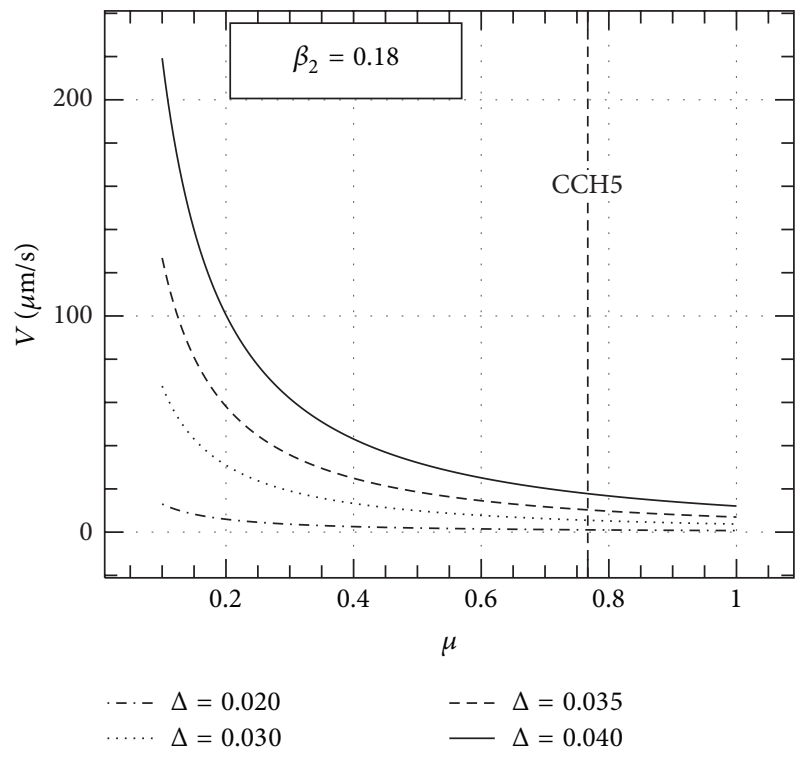

(a)

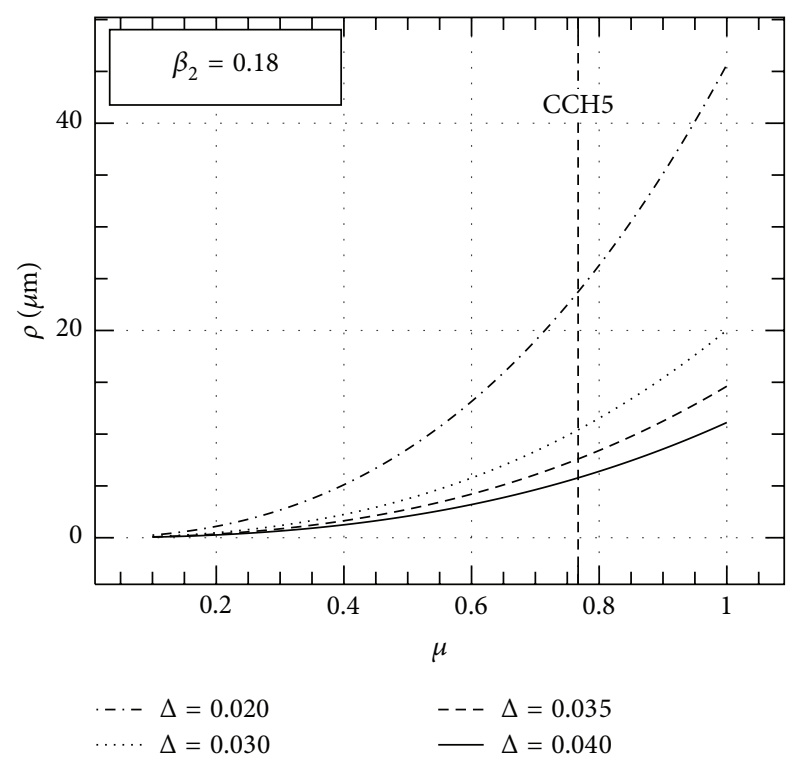

(b)

FIgURE 3: Observable quantities; (a) growth velocity $V$ from (21) and (b) tip curvature radius $\rho$ from (22) with $\widetilde{b}_{2}=1.6608$ as functions of the thermal diffusion anisotropy strength $\mu$ at different values of the dimensionless undercooling $\Delta$.

the growth direction. That is, most of the heat is removed sideways (along the $x$ direction) without increasing the

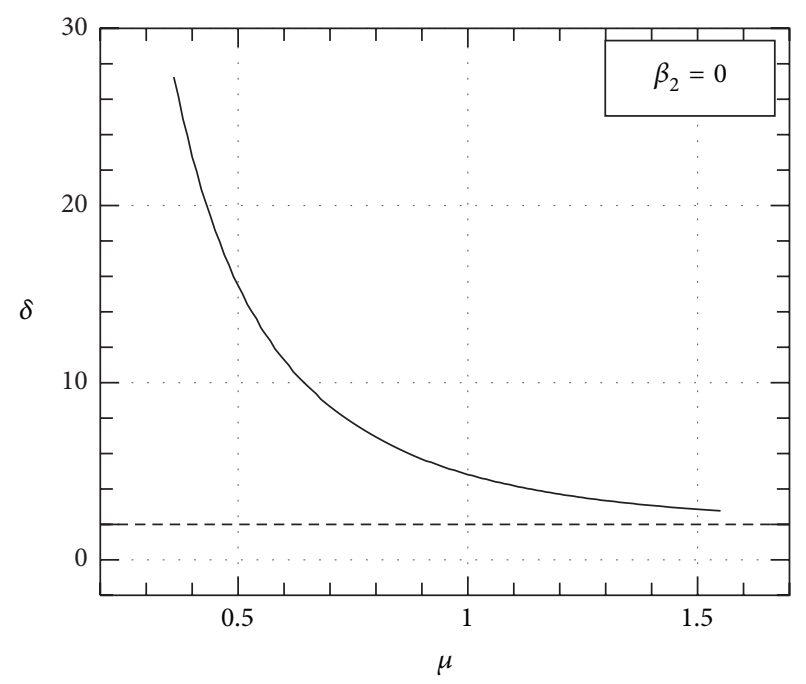

FIGURE 4: Derivative difference $\delta$ of $\dot{\phi}$ at the crossing point of the two lines of numerical integration as a function of the heat diffusion anisotropy strength $\mu$ (solid graph) in the case of isotropic surface tension $\left(\beta_{2}=0\right)$. The approximate asymptote at $\delta=2$ is marked by a dashed line.

temperature in front of the dendrite (in the $y$ direction), and the growth may proceed into cooler regions.

The numerical algorithm is based on a solution of (20) on two perpendicular lines in the complex plane. One line lies on the real axis and the other line is parallel to the imaginary axis. To obtain an analytic solution, the difference $\delta$ of the derivatives $\Psi^{\prime}$ on both lines is driven to zero at the crossing point. This is achieved by adjusting $\widetilde{b}_{2}$. This procedure leads to the correct eigenvalue in the case of isotropic diffusion [17]. In the case of isotropic surface tension $\left(b_{2}=0\right)$ and isotropic diffusion $(\mu=1)$, there is no solution [14]. But (18) with $b_{2}=0$ and $\mu<1$ can be solved for the derivative difference $\delta$ as a function of the heat diffusion anisotropy strength $\mu$. This is shown in Figure 4. The graph decays monotonically and it approximately approaches $\delta=2$ for $\mu>1.5$. The decay is nonexponential. Nevertheless, there are no zero-crossings. Thus, we find that heat diffusion anisotropy cannot stabilize the dendrite. If selection was supposed to happen analogously to the case of finite $\beta_{2}$, one would need a factor that becomes singular at $\mu=1$. Such a factor is not present in the local equation (18) at $b_{2}=0$. It is not clear how $\mu$ could take the stabilizing role of $\sigma$ in this model.

The numerical evidence is complemented by the fact that $\mu$ can be eliminated from (18) by rescaling, as shown at the end of the preceding section. In the case with $b_{2}=0$, this gives

$$
\frac{1+\Psi^{\prime \prime}}{\left(2\left(\Psi^{\prime}+\widetilde{t}\right)\right)^{3 / 2}}=\frac{\Psi}{2} .
$$

But this is the selection problem of the isotropic diffusion case with isotropic surface tension, known not to have a solution [14]. Since $\mu$ has completely scaled out of the equation, it cannot be seen, from a mathematical point of view, how 
the sole presence of $\mu<1$ could lead to a stable dendrite. Heat diffusion anisotropy obviously does not constitute a singular perturbation, which could break the degeneracy of Ivantsov's solution spectrum. In stark contrast to that, for nonzero $\sigma$, the problem is drastically changed by the capillary term in the Gibbs-Thomson condition (8).

\section{Conclusions}

In this study, we have investigated the influence of anisotropic diffusion on the (two-dimensional) selection problem in dendritic growth.

Our main results may be summarized in three statements. First, anisotropy of diffusion by itself is insufficient to select a stationary operating state for a close-to-parabolic needle crystal. Second, if there is anisotropy of surface tension in addition, we do find a solution to the selection problem, and we obtain analytic expressions for the selected velocity and tip radius as a function of the anisotropy ratio $\mu$ in the full $\mu$ range, containing just a single eigenvalue that has to be calculated numerically. Thirdly, we achieve a quantitative description and explanation of the phenomenon of "inverted growth."

\section{Conflict of Interests}

The authors declare that there is no conflict of interests regarding the publication of this paper.

\section{Acknowledgment}

Financial support of this work by the German Research Foundation (DFG) under Grant no. KA 672/10-1 is gratefully acknowledged.

\section{References}

[1] G. P. Ivantsov, "Temperature field around a spheroidal, cylindrical and acicular crystal growing in a supercooled melt," Doklady Akademii Nauk SSSR, vol. 58, pp. 567-569, 1947.

[2] M. Ben Amar and Y. Pomeau, "Theory of dendritic growth in a weakly undercooled melt," Europhysics Letters, vol. 2, no. 4, pp. 307-314, 1986.

[3] A. Dougherty and J. P. Gollub, "Steady-state dendritic growth of $\mathrm{NH}_{4} \mathrm{Br}$ from solution," Physical Review A, vol. 38, no. 6, pp. 3043-3053, 1988.

[4] E. R. Rubinstein and M. E. Glicksman, "Dendritic grown kinetics and structure," Journal of Crystal Growth, vol. 112, no. 1, pp. 84-96, 1991.

[5] M. Muschol, D. Liu, and H. Z. Cummins, "Surface-tensionanisotropy measurements of succinonitrile and pivalic acid: comparison with microscopic solvability theory," Physical Review A, vol. 46, no. 2, pp. 1038-1050, 1992.

[6] E. A. Brener, C. Hüter, D. Pilipenko, and D. E. Temkin, "Velocity selection problem in the presence of the triple junction," Physical Review Letters, vol. 99, no. 10, Article ID 105701, 2007.

[7] P. Bouissou and P. Pelcé, "Effect of a forced flow on dendritic growth," Physical Review A, vol. 40, no. 11, pp. 6673-6680, 1989.
[8] M. von Kurnatowski, T. Grillenbeck, and K. Kassner, "Selection theory of free dendritic growth in a potential flow," Physical Review E, vol. 87, Article ID 042405, 2013.

[9] M. von Kurnatowski and K. Kassner, "Scaling laws of free dendritic growth in a forced Oseen flow," Journal of Physics A: Mathematical and Theoretical, vol. 47, no. 32, Article ID 325202, 12 pages, 2014.

[10] P. Oswald, J. Bechhoefer, and A. Libchaber, "Instabilities of a moving nematic-isotropic interface," Physical Review Letters, vol. 58, no. 22, pp. 2318-2321, 1987.

[11] A. J. Simon and A. Libchaber, "Moving interface: the stability tongue and phenomena within," Physical Review A, vol. 41, no. 12, pp. 7090-7093, 1990.

[12] R. González-Cinca, L. Ramírez-Piscina, J. Casademunt et al., "Heat diffusion anisotropy in dendritic growth: phase field simulations and experiments in liquid crystals," Journal of Crystal Growth, vol. 193, no. 4, pp. 712-719, 1998.

[13] T. Börzsönyi, Á. Buka, and L. Kramer, "Effect of the anisotropic surface tension, crystallization kinetics, and heat diffusion on nonequilibrium growth of liquid crystals," Physical Review E, vol. 58, no. 5, pp. 6236-6245, 1998.

[14] E. A. Brener and V. I. Mel'nikov, "Pattern selection in twodimensional dendritic growth," Advances in Physics, vol. 40, no. 1, pp. 53-97, 1991.

[15] T. Fischaleck and K. Kassner, "Extending the scope of microscopic solvability: combination of the Kruskal-Segur method with Zauderer decomposition," Europhysics Letters, vol. 81, no. 5, Article ID 54004, 2008.

[16] C. Misbah, "Velocity selection for needle crystals in the 2-D one-sided model," Journal de Physique, vol. 48, no. 8, pp. 12651272, 1987.

[17] S. Tanveer, "Analytic theory for the selection of a twodimensional needle crystal at arbitrary Péclet number," Physical Review A, vol. 40, no. 8, pp. 4756-4769, 1989.

[18] T. Tóth-Katona, T. Börzsönyi, Z. Váradi et al., "Pattern formation during mesophase growth in a homologous series," Physical Review E, vol. 54, no. 2, pp. 1574-1583, 1996.

[19] F. Rondelez, W. Urbach, and H. Hervet, "Origin of thermal conductivity anisotropy in liquid crystalline phases," Physical Review Letters, vol. 41, no. 15, pp. 1058-1062, 1978.

[20] Å. Buka, T. Tóth-Katona, and L. Kramer, "Equilibrium shapes of a nematic-smectic-B liquid-crystal interface," Physical Review E, vol. 49, no. 6, pp. 5271-5275, 1994. 

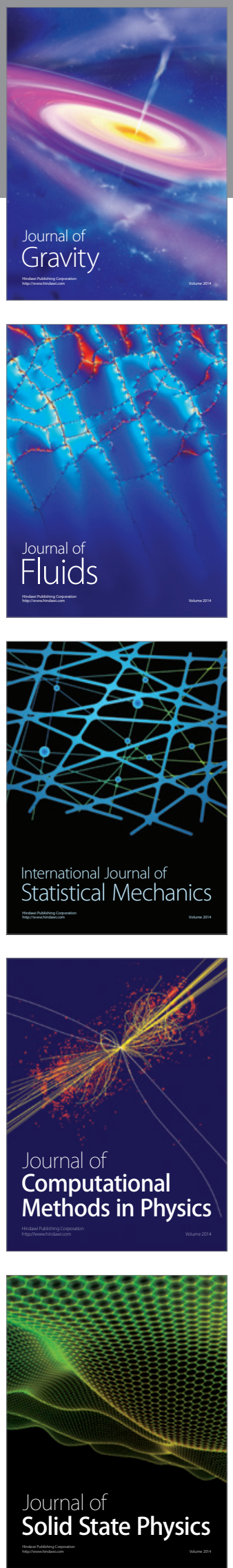

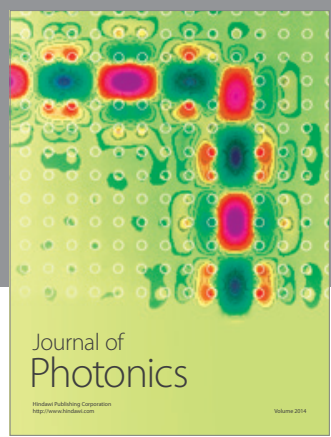

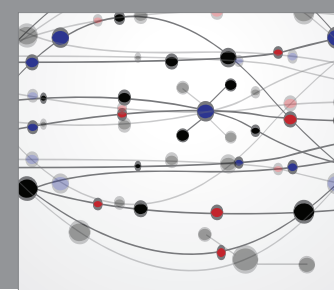

The Scientific World Journal

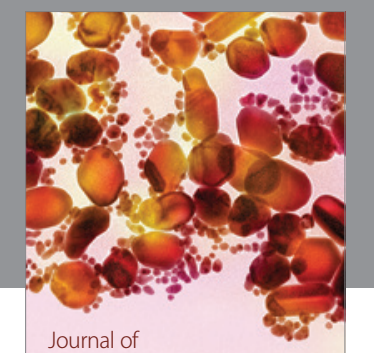

Soft Matter
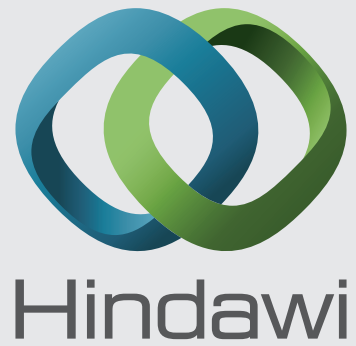

Submit your manuscripts at

http://www.hindawi.com
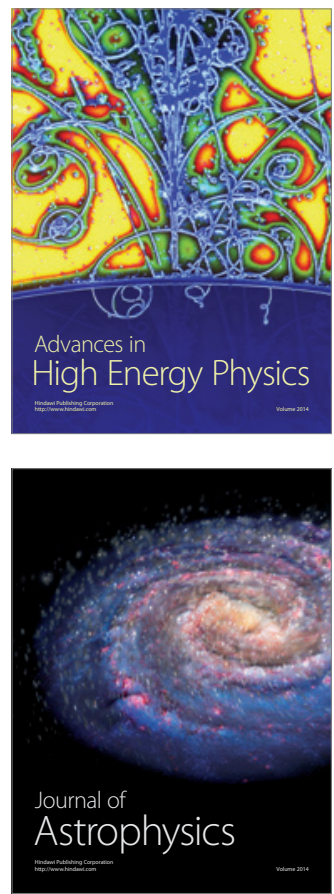
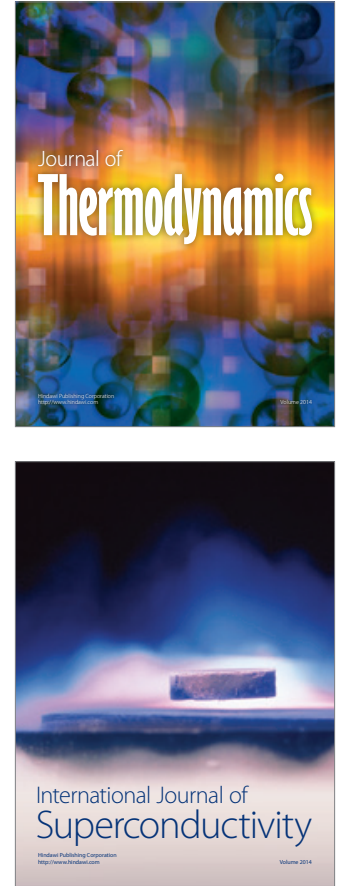
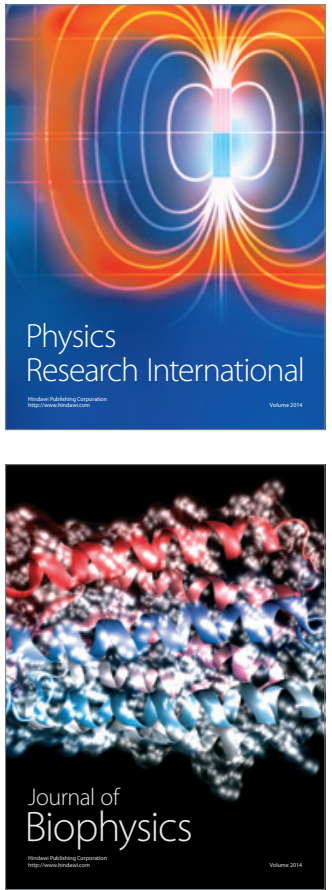
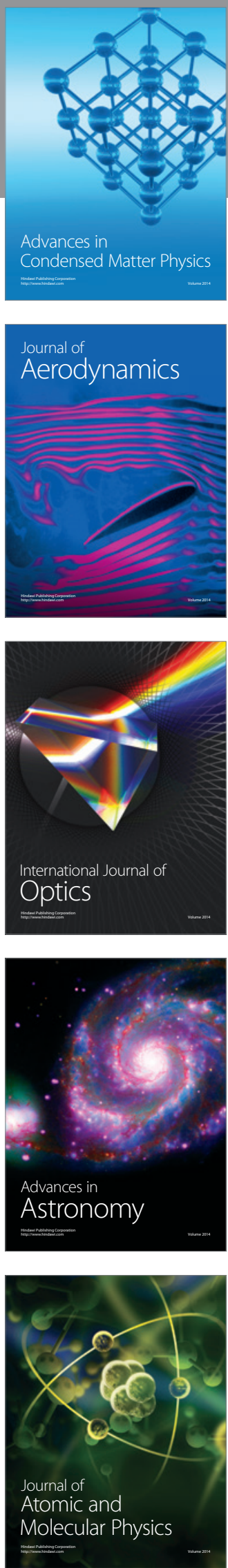